\title{
Research on Spatial Distribution Characteristics of Residential Homes with Rural Characteristics in China
}

\author{
Guoqiang Feng ${ }^{1, *}$ \\ ${ }^{1}$ School of Geomatics and Urban Spatial Informatics, Beijing University of Civil Engineering and Architecture, No.15 Yongyuan Rd., \\ Daxing District, Beijing, 102616, China
}

\begin{abstract}
In view of the current situation of the development of residential accommodation in rural areas in China, this paper starts from the spatial distribution and structural characteristics of residential accommodation with national characteristics, taking the national rural characteristic hostels as the research object, analyzes the spatial distribution characteristics of the characteristic hostels in the whole country by using the inverse distance weight method and Kriging spatial interpolation method, and visually expresses the development status of the characteristic hostels in the whole country by using the spatial thermal diagram. The experimental results show that the distribution of residential accommodation with rural characteristics in China has strong spatial agglomeration characteristics. Residential accommodation with rural characteristics in China is generally positively correlated with population density, occupied area and GDP in various regions. Relying on the beautiful natural environment and simple human resources, the development of the rural residents' lodging has made the rural residents' lodging itself a tourism attraction, transforming the disadvantages into advantages, driving the development of rural tourism and related industries, stimulating the employment of farmers and promoting cultural revival and the prosperity of the common people.
\end{abstract}

\section{Introduction}

In recent years, the development of residential accommodation in mainland China has continued the concept of residential accommodation in Japan and Taiwan. Family hotels and farmhouse entertainment are the embryonic forms of residential accommodation development, providing some short-term housing and leisure services for people living or working in cities. With the increasing development of the home stay industry, some restrictions have been gradually added on the original basis to distinguish home stay from traditional hotels [1]. In "Basic Requirements and Evaluation of Tourist Homestay" (LB/T 065-2017), the National Tourism Administration defines tourist homestay as "small accommodation facilities for tourists to experience local nature, culture, production and life style by using local idle resources and participating host people in reception" [2]. Homestay does not have the value advantage of economies of scale in a certain sense, but Chinese rural characteristic homestay provides tourists with different experiences through local high-quality landscape, personalized service and characteristic leisure activities, and pays more attention to the living environment, service quality and interaction with local activities in terms of business philosophy. To sum up, China's rural residential accommodation not only provides necessary basic services such as catering and accommodation, but also provides the life experience of local residents [3].

With the vigorous development of China's economy, the development of rural tourism has been highly valued by governments at all levels. In order to pursue the demand of rural original ecological tourism, China's rural tourism service has developed into a new industry in the tourism service industry. In response to the "the belt and road initiative" policy, promote the comprehensive development of rural economy, culture, science and technology, life and other aspects, combined with the wide application of modern science and technology. Based on the current development trend of rural residential accommodation industry in China, this paper forecasts the development trend of rural characteristic residential accommodation, which has certain practical significance for promoting its reasonable spatial distribution and the formation of competition pattern, so as to promote the sustainable development of rural revitalization.

\footnotetext{
* Corresponding author: fgq15600851912@163.com
} 


\section{Materials and Methods}

\subsection{Research Data}

The screening criteria of Chinese rural residential accommodation is the key to the establishment of the national database of rural residential accommodation. Based on the understanding of the concept and characteristics of residential accommodation, this paper defines Chinese rural residential accommodation, that is, rural residential accommodation with the following three characteristics can be included in the national database of rural residential accommodation.

(1) It has a good living environment and is located in traditional Chinese villages or surrounding areas.

(2) It has unique rural features and can meet tourists' vacation and leisure needs.

(3) It has the characteristics of rural regional culture, can show local flavor and highlight local culture.

This paper focuses on the table structure design and optimization of the storage of information such as natural geography, cultural landscape, longitude and latitude coordinates, real-life photos, online time, address and contact information of Chinese rural characteristic hostels. The details are shown in Table 1 below:

Tab.1 Data type and source

\begin{tabular}{|c|c|c|}
\hline Data Name & Field Attribute & Data Source \\
\hline $\begin{array}{c}\text { Rural characteristic } \\
\text { home stay } \\
\text { Population Data }\end{array}$ & $\begin{array}{c}\text { data, name, address, } \\
\text { telephone, etc. }\end{array}$ & under the east fence \\
\hline $\begin{array}{c}\text { Population Density } \\
\text { Data }\end{array}$ & $\begin{array}{c}\text { Sixth National } \\
\text { Population Census }\end{array}$ \\
\hline
\end{tabular}

(1) Data on residential accommodation with rural characteristics. China's most beautiful rural tourism service system (under the east fence) provides a total of 1620 residential accommodation site data, which basically covers all provinces and cities in the country in terms of spatial distribution. The data have good regional representation.

(2) Population data. In order to analyze the relationship between the number of residential accommodation and the number of population, this experiment uses the data of China's 6th National Population Census (2010).

(3) Economic status data. In order to analyze the relationship between the number of residential accommodation and the economic status of various regions, the experiment used the 2018 GDP data of the National Bureau of Statistics.

\subsection{Research Methods}

\subsection{1, Inverse distance weight method}

The interpolation unit value is obtained by averaging each sampling point in the adjacent area by using the inverse distance weight (IDW) method [4]. The distance between the interpolation point and the sample point is taken as the weight for weighted average, the spatial distance between coordinate points of rural characteristic residential accommodation is calculated, and the spatial distribution characteristics of large data of rural characteristic residential accommodation are studied.

\subsection{2, Kriging method}

Kriging method uses spatial statistics as the theoretical basis. Based on the data of some known sample points in the finite neighborhood of the unknown sample points, a linear unbiased optimal estimation of the unknown sample points is carried out after considering the shape, size and spatial orientation of the sample points, the spatial positional relationship with the unknown sample points, and the structural information provided by the variogram. [5].

\subsection{3, Ordinary least square method}

Ordinary Least Square (OLS) is the most widely used parameter estimation method, which requires the least model conditions, that is, to minimize the sum of squares of the distances from all observed values on the scatter plot to the regression straight line. It can be used to process a set of measured data, find the dependence relationship between variables from the measured data [6], and use linear regression to model the relationship between a dependent variable and a set of explanatory variables to obtain the prediction results.

\section{Results \& Discussion}

\subsection{Distribution characteristic analysis}

From the perspective of spatial analysis visualization, the distribution characteristics of residential accommodation with Chinese rural characteristics are analyzed more intuitively by means of thermodynamic diagram, and interpolation is carried out by IDW and Kriging methods. The results are shown in Figs. 1 and 2. With different colors and brightness, thermodynamic diagrams reflect the spatial differences of the described data. The closer to red, the higher the data density, and the closer to blue, the lower the data density. The thermodynamic diagram of this paper is divided into 10 levels. The number of characteristic hostels is converted to different density value intervals, with the highest regional value being 10 and the lowest regional value being 1 . 


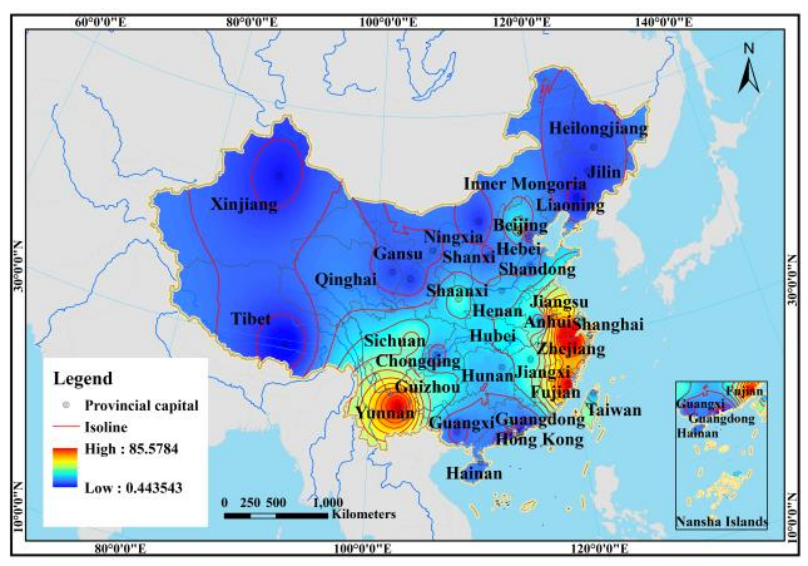

Fig.1 IDW spatial interpolation analysis distribution map of China's rural characteristic B\&B

Through IDW interpolation analysis method, the density distribution map of residential accommodation gathering areas with rural characteristics (Fig. 1) is obtained. The overall distribution has strong spatial clustering characteristics. The three high-density areas are Yunnan Province, Zhejiang Province and Fujian Province respectively. The local distribution pattern of each province and city is radial, spreading around the provincial capital to the surrounding towns [7].

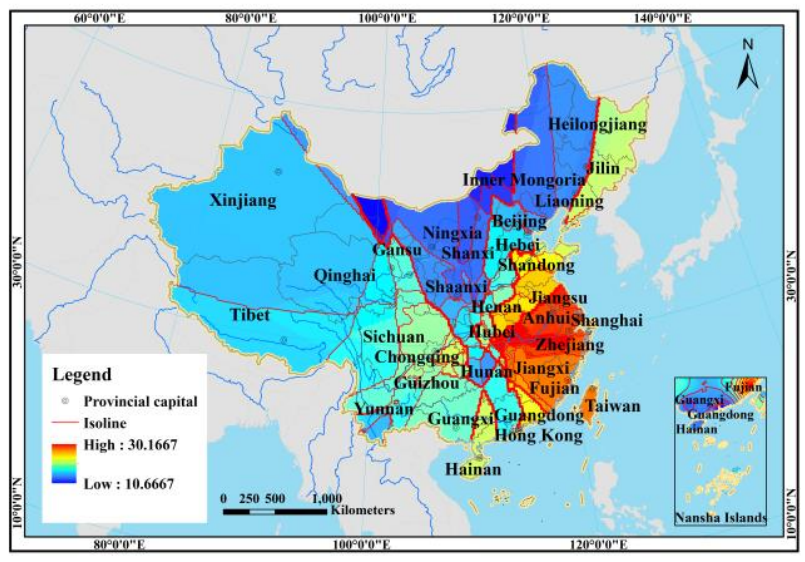

Fig.2 Kriging spatial interpolation analysis Chinese country characteristics $\mathrm{B} \& \mathrm{~B}$ distribution

At present, the residential accommodation with rural characteristics in China is distributed all over the country. The time sequence of the development of residential accommodation in rural areas is a gradual process from the south to the north or from the coastal areas to the inland. Using Kriging interpolation method to analyze the distribution characteristics of rural residential accommodation in China, it is concluded that there are many tourist resorts and scenic spots in coastal cities, and the number of rural residential accommodation is significantly more than that in other areas.

\subsection{Correlation analysis}

The provinces and autonomous regions with more residential accommodation in rural areas of China have relatively developed economies and high population density. On the contrary, in provinces and regions with backward economy and low population density, the distribution of residential accommodation in rural areas in China is also relatively small. Therefore, the least square method model is used to analyze the correlation between the residential data of rural residents in China and the population, occupied area and GDP data of various provinces and cities to find the best match. The results are shown in Figure 3.

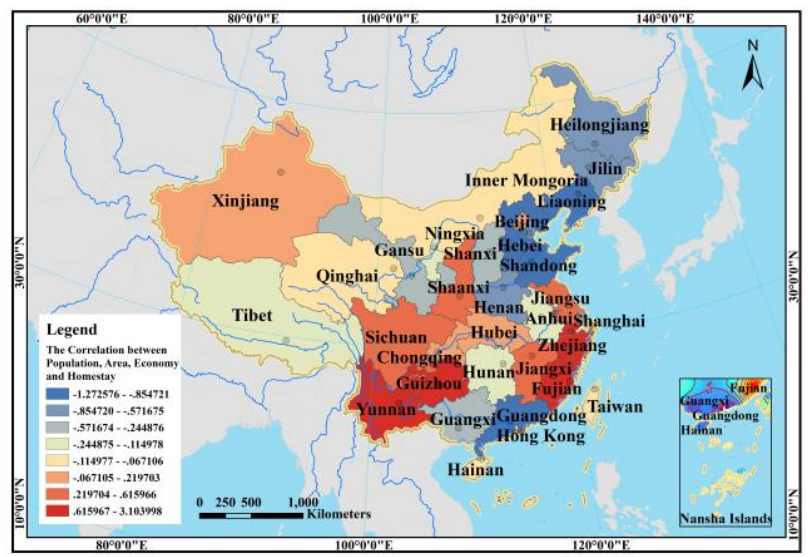

Fig. 3 Analysis of correlation between rural residents' lodging and population, area, economic of various provinces in China by least square method

The distribution of residential accommodation with rural characteristics in China has a positive correlation with the population, occupied area and GDP data of various provinces and cities. Among them, the distribution of residential accommodation with rural characteristics in Zhejiang, Yunnan, Fujian and Guizhou provinces has the highest correlation with the population, occupied area and GDP data. The residual values of 3.103998, 2.476107, 2.100371 and 0.802031 respectively. It shows that these areas have large population mobility, developed economy and small GDP, and the fault tolerance rate of rural residents' accommodation development is relatively low.

There is also a certain correlation between rural characteristic hostels and population density in northwest and central regions. The average residual value of 0.357078 and standard deviation of 0.190003 . This shows that the population density and the proportion of rural residents' accommodation distribution in these areas are relatively balanced, the area is vast, and the economy is relatively backward compared with other areas. Therefore, rural residents' accommodation should be vigorously developed.

The rural characteristic residential accommodation in the three northeastern provinces, some central areas and southern areas is negatively correlated with the population density. The average residual value of- 0.442707 and standard deviation of 0.381548 . This shows that these 
areas have high population density and relatively developed economy, but the distribution of residential accommodation in villages is very small. Therefore, there is still a lot of market space for the development of residential accommodation with rural characteristics in these areas.

\section{Results \& Discussion}

Through the statistical analysis of the spatial-temporal data of Chinese rural residential accommodation and the analysis of the spatial thermodynamic diagram, the following conclusions are drawn:

(1) Villagers' lodges in villages and towns in China have distinct spatial agglomeration characteristics, which are mainly concentrated in Yunnan, Fujian and Zhejiang provinces on the macro level.

(2) The local distribution pattern of each province and city is radial, spreading around the provincial capital to the surrounding towns;

(3) The distribution of residential accommodation is positively correlated with the population density, occupied area and GDP in each region.

According to the above research results, it shows that the development space of rural residents' hostels in China is still very sufficient, especially in the three northeastern provinces and the northwest region, which have rich tourism resources and are more suitable for vigorously developing rural residents' hostels. It is suggested that priority should be given to developing rural residents' hostels located close to tourist attractions in this region. However, compared with the southern region, the northern region also has certain market space and can make reasonable choices. In the southern region, Guangxi, Guangdong, Hunan and Anhui provinces can be considered. The geographical conditions are superior, with different characteristics of rural features and regional cultural characteristics, and the population capacity is large. It is worth developing residential accommodation for villagers in villages.

With the development of personalized tourism, many towns with historical and cultural backgrounds (such as Xi'an) will certainly be favored by tourists to build residential accommodation. This paper reveals the spatial distribution, agglomeration form, development trend and competition pattern of residential accommodation with Chinese rural characteristics, with a view to forming a conclusion with practical guiding significance.

\section{Acknowledgments}

This work was financially supported by The National Key Research and Development Program of China (2018YFC0706003) and the Pyramid Talent Training Project of Beijing University of Civil Engineering and Architecture (21082717008), and funded by the Beijing Key Laboratory of Urban Spatial Information Engineering (2020216).

\section{References}

1. Guo, Z., (2017) Between Residential Buildings and Hotels: Research on Homestay Design. Kunming University of Technology.

2. Chen X.L., (2019) Spatial pattern and influencing factors of residential accommodation in Sanya. Hunan Normal University.

3. Hou Y.X., Li X., Wu Z.J., (2017) Research progress on residential accommodation in China. Journal of Guangxi Institute of Economic Management Cadres, 29 (04): 60-66.

4. Ding X., Ai S.T., Wang Z.M., Hao W.F., Tan Zi., (2019) Study on surface elevation variations of Arctic Australian Lov é breen glacier based on RTK-GPS. Polar Research, 31 (03): 246-257.

5. Li J.X., Li C.K., Yin Z.H., (2013) Kriging interpolation method based on ArcGIS and its application. Surveying and Mapping Bulletin, (9): 87-90.

6. Liang X.O., Hu R., (2011) High-dimensional target reduction algorithm based on least square method. Software Guide, 10 (08): 39-42.

7. Mou Y., (2017) Spatial Distribution Characteristics and Investigation Zoning of China's Important Agricultural Cultural Heritage. Hubei University. 